\title{
Sharing a quantum secret without a trusted party
}

\author{
Qin Li $^{1,2, *}$, Dong Yang Long ${ }^{1}$, W. H. Chan ${ }^{2}$, Dao Wen Qiu ${ }^{1}$ \\ ${ }^{1}$ Department of Computer Science, Sun Yat-sen University, Guangzhou 510006 China \\ ${ }^{2}$ Department of Mathematics, Hong Kong Baptist University, Kowloon, Hong Kong, China
}

\begin{abstract}
In a conventional quantum $(k, n)$ threshold scheme, a trusted party shares a secret quantum state with $n$ participants such that any $k$ of those participants can cooperate to recover the original secret, while fewer than $k$ participants obtain no information about the secret. In this paper we show how to construct a quantum $(k, n)$ threshold scheme without the assistance of a trusted party, who generates and distributes shares among the participants. Instead, each participant chooses his private state and contributes the same to the determination of the final secret quantum state.
\end{abstract}

Keywords Quantum secret sharing · Quantum cryptography · Quantum information processing ·

Quantum communication

PACS 03.67.Dd

\section{Introduction}

Suppose that $n$ shareholders who are not mutually trusted want to share a password, with which they can open the vault and get access to some confidential documents of the company. It should be done in such a way that any $k$ of those shareholders have the ability to reconstruct the password, while fewer than $k$ shareholders cannot. The solutions vary under two different situations. If there exists a trusted

${ }^{*}$ Corresponding author: Qin $\mathrm{Li}$

E-mail address: liqin805@163.com (Q. Li) 
party who generates and distributes suitable shares among the shareholders, then the problem could be addressed by classical $(k, n)$ threshold schemes independently introduced by Blakley [1] and Shamir [2]. And if there does not exist such a party trusted by all of the shareholders, then classical $(k, n)$ threshold schemes without the assistance of any trusted party came to rescue [3] 5$]$.

With the emergence of quantum computation and quantum communication, it is natural to consider the quantum counterparts of secret sharing schemes. Hillery et al. showed how to implement a classical threshold scheme using Greenberger-Horne-Zeilinger (GHZ) states in the presence of eavesdroppers, and also showed how to share an unknown qubit between two participants such that only the collaboration of two participants could reconstruct the original qubit [6]. Karlsson et al. presented a secret sharing scheme based on two-particle quantum entanglement, in which quantum information is sent from a sender Trent to Alice and Bob so that both persons are needed to obtain the information, and showed that it could be extended to a special quantum $(k, n)$ threshold scheme based on multi-particle entangled states [7]. Cleve et al. gave an efficient construction of more general quantum $(k, n)$ threshold schemes, where a trusted party can share an unknown quantum state with $n$ participants such that $k$ participants are necessary and sufficient to reconstruct the original secret quantum state [8]. Thereafter quantum secret sharing has been an active research field and many quantum secret sharing schemes using various techniques have been proposed [9[20].

Whereas most previous quantum $(k, n)$ threshold schemes have been considered with the assistance of a trusted party [6] 20], here we consider the problem of sharing a quantum secret without having a trusted party. Actually, for lots of applications such as conference key agreement and distributed computing with faulty processors, quantum secret sharing without a trusted party is a powerful tool. Also, as referred by Ingemarsson and Simmons $[3$, the situation that there is nobody trusted by all of the participants is more common in commercial and/or international applications. In this case, previously proposed quantum secret schemes do not play the role well. Therefore, in this paper we give a basic construction for sharing a quantum secret in the absence of a trusted party and illustrate its feasibility mainly by improving the quantum $(k, n)$ threshold scheme presented by Cleve et al. 8 , to eliminate the need of the trusted party. 
The novelty is that a trusted party is unnecessary and each participant acts for his own benefit.

\section{Quantum $(k, n)$ threshold schemes without a trusted party}

In this section, we show how to solve the problem of sharing a quantum secret without the aid of any trusted party, drawing ideas from classical counterparts 3 5. The problem could be stated more clearly as follows:

- all of the participants choose their own private quantum states, which must be made available in sequence for the final secret quantum state to be generated;

- all of the participants contribute identically to determining the quantum secret;

- the participants share the secret quantum state in a way that the collaboration of any $k$ out of $n$ participants can recover the secret quantum state, but the collaboration of any $k-1$ or fewer participants obtain no information about it.

Such a scheme is called a quantum $(k, n)$ threshold scheme without a trusted party. In such a scheme, each participant acts for his own benefit and need not trust a single party unless at least $k$ participants work together. In addition, notice $n<2 k$ for a quantum $(k, n)$ threshold scheme without a trusted party, which is guaranteed by quantum no-cloning theorem [21].

The basic method used for constructing a quantum $(k, n)$ threshold scheme without a trusted party is introduced in the following.

(1) Each participant $P_{i}(i=0,1, \cdots, n-1)$ randomly selects his private quantum state $\rho_{i}$ which can be a component of the quantum secret, or belong to the same domain as that of the secret quantum state to be reconstructed. The specific form of the quantum secret can vary with different applications and should be defined before implementing the protocol.

(2) $P_{i}$ acts as a trusted party and splits his private quantum state $\rho_{i}$ into $n$ shares $\rho_{i j}(j=0,1, \cdots, n-1)$ using a conventional quantum $(k, n)$ threshold scheme such as [8], and then sends each share $\rho_{i j}$ to the participant $P_{j}$ in sequence (particularly $P_{i}$ keeps $\rho_{i i}$ ). 
(3) When $P_{i}$ has received the share $\rho_{j i}$ from the participants $P_{j}$, he can do nothing or implement corresponding operations on $\rho_{j i}$ and his quantum registers. Note that such operations are agreed before the protocol and vary with the conventional quantum $(k, n)$ threshold schemes used. If $P_{i}$ has received the shares $\rho_{j i}(j=0,1, \cdots, n-1)$ and implemented the corresponding operations by some time (the time limit is agreed before the protocol), he announces that he has completed his actions; else if $P_{i}$ has not get the share from some party $P_{l}$, he also reveals this fact.

(4) If at least $k$ parties have not received the shares from some party $P_{l}$ before the time limit, they consider $P_{l}$ a quitter and not a real player. If the number of quitters is not exceeding $n-k$, the protocol cannot be affected and just the value of $n$ decreases; otherwise the protocol aborts. After all of the real participants have announced, any $k$ participants can cooperate to obtain the final secret quantum state $\rho_{s}$ which each participant's private state $\rho_{i}$ contributes equally to by a preconcerted way, while less than $k$ participants obtain no information about $\rho_{s}$ except their own private states.

Note that we should assume that each participant must logically be willing to share the private quantum state chosen by himself with other participants and contribute the same to determining the final secret quantum state. Otherwise a malicious participant who sends an improper quantum states can make $k$ participants reconstruct a wrong quantum secret and thus make the protocol abort, even though doing such things is of no use for him. In addition, the security of such a quantum $(k, n)$ threshold scheme without having a trusted party largely depends on the conventional quantum $(k, n)$ threshold scheme used by each participant to protect his private state among the other participants before the recovery phase.

\section{Concrete quantum $(k, n)$ threshold schemes without a trusted party}

In order to clarify the feasibility of the construction of a quantum $(k, n)$ threshold scheme without a trusted party introduced by us, we give a couple of schemes. Besides, it is only necessary for us to consider real players' actions, since the construction method has ensured that if at most $n-k$ players drop out midway, the protocol will not be affected.

one scheme can be achieved as follows just by utilizing the operations included in a conventional quantum $(k, n)$ threshold scheme such as that in [6] 8 : each participant shares the private state chosen by 
himself with the other participants using a conventional quantum $(k, n)$ threshold scheme; if a participant has obtained the shares and performed accordingly, he makes an announcement that his task has been finished; then any $k$ players recover each participant's private state using the conventional recovery procedure; and such $k$ players obtain the final secret from all of the recovered private states by implementing the agreed operations. Obviously, the processes of this scheme can accord with that of the proposed construction method and the trusted party is removed indeed. Nevertheless, the conventional recovery procedure has to be implemented for $n$ times and then the negotiated operations should be applied on $n$ private states to obtain a secret.

Another concrete scheme is based on the quantum $(k, n)$ threshold scheme presented by Cleve et al. [8]. We improve it to remove the use of the trusted party. The improved scheme can allow all of the participants to choose their own private state and have the same influence on determining the final quantum secret.

Note $n<2 k$ and that the dimension of each share can be bounded above by $2 \max (2 k-1, m)$ through efficient quantum operations, where $m$ is the dimension of the private quantum state to be encoded, the same as that in $[\underline{8}$. In addition, we just need to consider the special case where $n=2 k-1$, since a quantum $(k, n)$ threshold scheme with $n<2 k-1$ can be obtained by discarding $2 k-1-n$ shares from any $(k, 2 k-1)$ threshold scheme with $n>k[\underline{8}$. Our improved scheme without the aid of the trusted party includes three parts: scheme setup, secret quantum state generation, and secret quantum state reconstruction.

\subsection{Scheme setup}

Given $k, n$ and $m$, find a suitable prime $q$ satisfying $\max (n, m) \leq q \leq 2 \max (n, m)$ (which is always possible according to Bertrand's postulate [22]) and set a finite field $\mathbf{F}=\mathbf{Z}_{q}$. For $i=0,1, \cdots, n-1$, let $c_{i}=\left(c_{i, 0}, c_{i, 1}, \cdots, c_{i, k-1}\right) \in \mathbf{F}^{k}$, define the polynomial as $p_{c_{i}}(t)=c_{i, 0}+c_{i, 1} t+\cdots+c_{i, k-1} t^{k-1}$, and let $x_{i} \in \mathbf{F}$ and each $x_{i}$ should be different from each other. Then a $q$-ary quantum state which is defined on basis states $\left|s_{i}\right\rangle\left(s_{i} \in \mathbf{F}\right)$ could be encoded by the linear mapping as

$$
\left|s_{i}\right\rangle \rightarrow \sum_{c_{i} \in \mathbf{F}^{k}, c_{i, k-1}=s_{i}}\left|p_{c_{i}}\left(x_{0}\right), p_{c_{i}}\left(x_{1}\right), \cdots, p_{c_{i}}\left(x_{n-1}\right)\right\rangle .
$$


Define the resulting state of adding a $q$-ary quantum basis state $\left|s_{i}\right\rangle$ to the quantum register in a $q$-ary quantum basis state $\left|s_{j}\right\rangle$ as $\left|s_{i}+s_{j}\right\rangle\left|s_{i}\right\rangle\left(s_{i}, s_{j} \in \mathbf{F}\right)$, which can be encoded by the linear mapping as

$$
\begin{aligned}
& \sum_{c_{i}, c_{j} \in \mathbf{F}^{k}}\left|p_{c_{i}}\left(x_{0}\right)+p_{c_{j}}\left(x_{0}\right), \cdots, p_{c_{i}}\left(x_{n-1}\right)+p_{c_{j}}\left(x_{n-1}\right)\right\rangle\left|p_{c_{i}}\left(x_{0}\right), \cdots, p_{c_{i}}\left(x_{n-1}\right)\right\rangle . \\
& c_{i, k-1}=s_{i}, c_{j, k-1}=s_{j}
\end{aligned}
$$

Also define the operation applying an invertible $l \times l$ matrix $M$ to a sequence of $l$ quantum registers as applying the mapping

$$
\left|\left(y_{0}, y_{1}, \cdots, y_{l-1}\right)\right\rangle \rightarrow\left|\left(y_{0}, y_{1}, \cdots, y_{l-1}\right) M\right\rangle
$$

For $z_{0}, z_{1}, \cdots, z_{l-1} \in \mathbf{F}$, introduce the $l \times l$ Vandermonde matrix $\left[V_{l}\left(z_{0}, z_{1}, \cdots, z_{l-1}\right)\right]_{i j}=z_{j}^{i}(i, j \in$ $\{0,1, \cdots, l-1\})$. And notice that

$$
\begin{aligned}
& \left|\left(c_{i, 0}, c_{i, 1}, \cdots, c_{i, l-1}\right) V_{l}\left(z_{0}, z_{1}, \cdots, z_{l-1}\right)\right\rangle \\
= & \left|p_{c_{i}}\left(z_{0}\right), p_{c_{i}}\left(z_{1}\right), \cdots, p_{c_{i}}\left(z_{l-1}\right)\right\rangle,
\end{aligned}
$$

where $c_{i}=\left(c_{i, 0}, c_{i, 1}, \cdots, c_{i, l-1}\right) \in \mathbf{F}^{l}$.

In addition, suppose that each participant $P_{i}(i=0,1, \cdots, n-1)$ owns a local quantum register $R_{i}$ and $n$ common quantum registers $C_{i, j}(j=0,1, \cdots, n-1)$ and every register is in an initial state $|0\rangle(0 \in \mathbf{F})$.

\subsection{Secret quantum state generation}

In this phase, $P_{i}(i=0,1, \cdots, n-1)$ will adopt a method of encoding by employing a polynomial of degree $k-1$ denoted as $p_{c_{i}}(t)=c_{i, 0}+c_{i, 1} t+\cdots+c_{i, k-1} t^{k-1}$, where $c_{i}=\left(c_{i, 0}, c_{i, 1}, \cdots, c_{i, k-1}\right) \in \mathbf{F}^{k}$.

$P_{i}$ randomly chooses and prepares his own private $q$-ary quantum state $\left|s_{i}\right\rangle\left(s_{i} \in \mathbf{F}\right)$ and uses the encoding denoted as (1) to obtain

$$
\sum_{c_{i} \in \mathbf{F}^{k}, c_{i, k-1}=s_{i}}\left|p_{c_{i}}\left(x_{0}\right), p_{c_{i}}\left(x_{1}\right), \cdots, p_{c_{i}}\left(x_{n-1}\right)\right\rangle .
$$


Note that the above operation implies that the quantum state $\left|s_{i}\right\rangle$ is split into $n$ shares which are stored in the quantum registers $C_{i, j}(j=0,1, \cdots, n-1)$. Then $P_{i}$ sends the share in the register $C_{i, j}$ to $P_{j}$ for $j=0,1, \cdots, n-1$ (including himself) sequently in an authenticated way.

When $P_{i}$ receives a share, he adds the received state to the quantum register $R_{i}$. And if $P_{i}$ has obtained the shares from all of the participants and implemented all the corresponding operations, he announces that his actions have been finished. After all of the participants have announced, it is not difficult to obtain that $n+n^{2}$ quantum registers $R_{0}, R_{1}, \cdots, R_{n-1}, C_{0,0}, C_{0,1}, \cdots, C_{n-1, n-1}$ are in a global state

$$
\begin{aligned}
& \sum_{c_{i} \in \mathbf{F}^{k}, c_{i, k-1}=s_{i}}\left|\sum_{i=0}^{n-1} p_{c_{i}}\left(x_{0}\right), \cdots, \sum_{i=0}^{n-1} p_{c_{i}}\left(x_{n-1}\right)\right\rangle \otimes_{i=0}^{n-1}\left|p_{c_{i}}\left(x_{0}\right), \cdots, p_{c_{i}}\left(x_{n-1}\right)\right\rangle . \\
& \text { for } i=0, \cdots, n-1
\end{aligned}
$$

Then, let the final agreed secret quantum state be $|s\rangle$ (for $s=\sum_{i} s_{i}=\sum_{i} c_{i, k-1}$ and $s \in \mathbf{F}$ ) if all the participants are required to provide $q$-ary quantum basis states before the scheme. In this case, we shall see that the final secret can be recovered using the conventional recovery procedure only once. However, if players can be allowed to provide a superposition of basis states, their private states may entangle with the resulting state after performing operations on them. So the final secret state should be defined in a different way, such as a superposition of states $\left|\sum_{i=0}^{n-1} s_{i}\right\rangle \otimes_{i=0}^{n-1}\left|s_{i}\right\rangle\left(s_{i} \in \mathbf{F}\right)$ or measuring the superposition state using the computational basis in $F^{n+1}$ and taking the collapsed state $\left|\sum_{i=0}^{n-1} s_{i}\right\rangle$ in the first register as a final secret. The form of the final secret can vary with the specific applications and should be negotiated before implementing the scheme.

\subsection{Secret quantum state reconstruction}

In this part, we show that the collaboration of any $k$ participants can reconstruct the agreed secret quantum state $|s\rangle$ by the following steps. Suppose that the first $k$ participants, namely $P_{0}, P_{1}, \cdots, P_{k-1}$, gather together and want to recover the secret, so we obtain the information about the fist $k$ quantum registers (that is, $R_{0}, \cdots, R_{k-1}$ ) and $k n$ common quantum registers (namely, $C_{i, j}$ for $i=0, \cdots, n-1$ and $j=0, \cdots, k-1)$. 
(1) Apply $V_{k}\left(x_{0}, x_{1}, \cdots, x_{k-1}\right)^{-1}$ which represents the inverse of $V_{k}\left(x_{0}, x_{1}, \cdots, x_{k-1}\right)$ to the first $k$ quantum registers $R_{0}, R_{1}, \cdots, R_{k-1}$. Then the global state of the $n+n^{2}$ quantum registers is

$$
\begin{aligned}
& \quad \sum_{c_{i} \in \mathbf{F}^{k}, c_{i, k-1}=s_{i}}\left|\sum_{i=0}^{n-1} c_{i, 0}, \cdots, \sum_{i=0}^{n-1} c_{i, k-1}\right\rangle\left|\sum_{i=0}^{n-1} p_{c_{i}}\left(x_{k}\right), \cdots, \sum_{i=0}^{n-1} p_{c_{i}}\left(x_{n-1}\right)\right\rangle \otimes_{i=0}^{n-1}\left|p_{c_{i}}\left(x_{0}\right), \cdots, p_{c_{i}}\left(x_{n-1}\right)\right\rangle . \\
& \text { for } i=0, \cdots, n-1
\end{aligned}
$$

(2) Shift the first $k$ quantum registers by one to the right in sequence by setting $R_{0}, R_{1}, \cdots, R_{k-1}$ to $R_{k-1}, R_{0}, \cdots, R_{k-2}$. At this time, the global state of the $n+n^{2}$ quantum registers is

$$
\begin{gathered}
\sum_{c_{i} \in \mathbf{F}^{k}, c_{i, k-1}=s_{i}}\left|\sum_{i=0}^{n-1} c_{i, k-1}, \sum_{i=0}^{n-1} c_{i, 0}, \cdots, \sum_{i=0}^{n-1} c_{i, k-2}\right\rangle\left|\sum_{i=0}^{n-1} p_{c_{i}}\left(x_{k}\right), \cdots, \sum_{i=0}^{n-1} p_{c_{i}}\left(x_{n-1}\right)\right\rangle \otimes_{i=0}^{n-1}\left|p_{c_{i}}\left(x_{0}\right), \cdots, p_{c_{i}}\left(x_{n-1}\right)\right\rangle \\
\text { for } i=0, \cdots, n-1 \\
=\quad|s\rangle\left|\sum_{i=0}^{n-1} c_{i, 0}, \cdots, \sum_{i=0}^{n-1} c_{i, k-2}\right\rangle\left|\sum_{i=0}^{n-1} p_{c_{i}}\left(x_{k}\right), \cdots, \sum_{i=0}^{n-1} p_{c_{i}}\left(x_{n-1}\right)\right\rangle \otimes_{i=0}^{n-1}\left|p_{c_{i}}\left(x_{0}\right), \cdots, p_{c_{i}}\left(x_{n-1}\right)\right\rangle . \\
\sum_{c_{i} \in \mathbf{F}^{k}, c_{i, k-1}=s_{i}} \\
\text { for } i=0, \cdots, n-1
\end{gathered}
$$

If the state in $R_{0}$ is a basis state $|s\rangle$ (for some $s \in \mathbf{F}$ ), it can be the final secret quantum state and the recovery procedure has been done; otherwise we should continue the recovery procedure. Actually, a participant usually offers a superposition of basis states, so the register $R_{0}$ is entangled with the other registers, since in (8) the value of $|s\rangle$ can be determined by any of the kets

$$
\left|\sum_{i=0}^{n-1} c_{i, 0}, \cdots, \sum_{i=0}^{n-1} c_{i, k-2}\right\rangle\left|\sum_{i=0}^{n-1} p_{c_{i}}\left(x_{k}\right), \cdots, \sum_{i=0}^{n-1} p_{c_{i}}\left(x_{n-1}\right)\right\rangle \otimes_{i=0}^{n-1}\left|p_{c_{i}}\left(x_{0}\right), \cdots, p_{c_{i}}\left(x_{n-1}\right)\right\rangle .
$$

(3) Apply $V_{k-1}\left(x_{k}, x_{k+1}, \cdots, x_{n-1}\right)$ to the quantum registers $R_{1}, R_{2}, \cdots, R_{k-1}$ and add $R_{0} \cdot\left(x_{k+i-1}\right)^{k-1}$ to $R_{i}$ for all $i \in\{1,2, \cdots, k-1\}$. And the global state of the $n+n^{2}$ quantum registers is

$$
\begin{aligned}
& \sum_{c_{i} \in \mathbf{F}^{k}, c_{i, k-1}=s_{i}}|s\rangle\left|\sum_{i=0}^{n-1} p_{c_{i}}\left(x_{k}\right), \cdots, \sum_{i=0}^{n-1} p_{c_{i}}\left(x_{n-1}\right)\right\rangle\left|\sum_{i=0}^{n-1} p_{c_{i}}\left(x_{k}\right), \cdots, \sum_{i=0}^{n-1} p_{c_{i}}\left(x_{n-1}\right)\right\rangle \otimes_{i=0}^{n-1} \mid p_{c_{i}}\left(x_{0}\right), \cdots, p_{c_{i}}\left(x_{n}-(\mathbf{1}) \boldsymbol{)}\right), \\
& \text { for } i=0, \cdots, n-1
\end{aligned}
$$

(4) The first $k$ parties perform the similar operations that were applied to the registers $R_{0}, R_{2}, \cdots, R_{k-1}$ in steps (1), (2) and (3) of the state reconstruction phase on the registers $C_{i, 0}, C_{i, 1}, \cdots, C_{i, k-1}$ for $i=$ 
$0,1, \cdots, n-1$. Then the global state of the $n+n^{2}$ quantum registers should be

$$
\begin{aligned}
& \qquad \sum_{c_{i} \in \mathbf{F}^{k}, c_{i, k-1}=s_{i}}|s\rangle\left|\sum_{i=0}^{n-1} p_{c_{i}}\left(x_{k}\right), \cdots, \sum_{i=0}^{n-1} p_{c_{i}}\left(x_{n-1}\right)\right\rangle\left|\sum_{i=0}^{n-1} p_{c_{i}}\left(x_{k}\right), \cdots, \sum_{i=0}^{n-1} p_{c_{i}}\left(x_{n-1}\right)\right\rangle \\
& \text { for } i=0, \cdots, n-1
\end{aligned}
$$

$$
\otimes_{i=0}^{n-1}\left(\left|s_{i}\right\rangle\left|p_{c_{i}}\left(x_{k}\right), \cdots, p_{c_{i}}\left(x_{n-1}\right)\right\rangle\left|p_{c_{i}}\left(x_{k}\right), \cdots, p_{c_{i}}\left(x_{n-1}\right)\right\rangle\right)
$$

(5) Shift the registers possessed by the first participant $P_{0}$ (namely, $R_{0}, C_{0,0}, \cdots, C_{n-1,0}$ ) to be first $n+1$ registers. For $i=(0,1, \cdots, k-1)$, since there is a unique array $c_{i} \in \mathbf{F}^{k}$ with $c_{i, k-1}=s_{i}$ such that $p_{c_{i}}\left(x_{k+j-1}\right)=y_{i, j}$ for any $j \in\{1,2, \cdots, k-1\}$, the state denoted as Eq. (11) can be rewritten as

$$
\begin{aligned}
& |s\rangle \otimes_{i=0}^{n-1}\left|s_{i}\right\rangle \sum_{c_{i} \in \mathbf{F}^{k}, c_{i, k-1}=s_{i}}\left|\sum_{i=0}^{n-1} y_{i, 1}, \cdots, \sum_{i=0}^{n-1} y_{i, k-1}\right\rangle\left|\sum_{i=0}^{n-1} y_{i, 1}, \cdots, \sum_{i=0}^{n-1} y_{i, k-1}\right\rangle \\
& \text { for } i=0, \cdots, n-1
\end{aligned}
$$

$$
\otimes_{i=0}^{n-1}\left(\left|y_{i, 1}, \cdots, y_{i, k-1}\right\rangle\left|y_{i, 1}, \cdots, y_{i, k-1}\right\rangle\right)
$$

From the Eq. (12), if all the participants provide $q$-ary quantum basis states, the state in $R_{0}$ is $|s\rangle$ (for $s=\sum_{i} s_{i}=\sum_{i} c_{i, k-1}$ and $\left.s \in \mathbf{F}\right)$ and it can be the final agreed secret. If some players offer superposition states of basis states, the state of the first $n+1$ registers (that is, $R_{0}, C_{0,0}, \cdots, C_{n-1,0}$ ) is a superposition of the states $\left|\sum_{i=0}^{n-1} s_{i}\right\rangle \otimes_{i=0}^{n-1}\left|s_{i}\right\rangle\left(s_{i} \in \mathbf{F}\right)$ which can be defined as the final secret. We also can measure the superposition state in the computational basis in $F^{n+1}$ and the collapsed state $\left|\sum_{i=0}^{n-1} s_{i}\right\rangle$ in $R_{0}$ can be considered as a final secret. Note that the the sum of the measurement outcomes of $C_{0,0}, \cdots, C_{n-1,0}$ should equate the value of $R_{0}$. If not, there must be something wrong and the scheme should be carried out again. The specific definition of the final secret can be adapted to different applications and should be established before performing the scheme.

In summary, the above two schemes where the trusted party is removed demonstrate the feasibility of the construction method offered by us. But in the first scheme, the conventional recovery procedure needs to be implemented for $n$ times and then the agreed operations on $n$ private states are needed to gain a secret. In the second scheme, if all the participants are required to provide basis states, the final secret can be reconstructed using the conventional recovery procedure only once. Nevertheless, if participants 
are allowed to offer superposition states, the conventional recovery procedure should be employed for $n+1$ times to generate a secret. In addition, the security of these two scheme largely relies on the conventional quantum $(k, n)$ threshold scheme since each participant needs it to protect his private state among the other participants before the secret reconstruction phase.

\section{Conclusion}

In this paper, we have given a basic construction of quantum $(k, n)$ threshold schemes without a trusted party and illustrated its feasibility by offering two schemes. In contrast to previous presented quantum secret sharing schemes [6] 20], in which a trusted party is always needed to assist in generating and distributing shares among a group of participants, the schemes suggested in this paper require no trusted party and thus might widen the applicability of quantum threshold schemes to the situation where there is no single party trusted by all of the participants.

However, the construction method proposed needs the participants to be logically honest during the secret generation phase since they should provide a proper quantum state; otherwise a single participant can disrupt the whole scheme and not be detected until the secret reconstruction phase, even though doing so is of no use for him. Although it is not quite reasonable to make such an expectation, the main intention of this paper is to demonstrate the necessity and feasibility of sharing a quantum secret without a trusted party. Particularly, Michael Ben-Or recently suggested us that the techniques used in quantum multiparty computation [23, 24] may be exploited to propose quantum secret sharing schemes in the absence of a trusted party where less than half of the participants are not required to play honestly during the secret generation phase. This invaluable advice shall build a bridge between quantum secret sharing without the aid of a trusted party and quantum multiparty computing. How to utilize them comprehensively will be the further work.

Acknowledgement We would like to appreciate Guang Ping He and Michael Ben-Or for useful suggestions. We are also very grateful to the anonymous referee for constructive comments and suggestions. This work was sponsored by the Faculty Research (Grant No. FRG2/08-09/070) Hong Kong Baptist University. 


\section{References}

[1] Blakley, G. R.: Safeguarding cryptographic keys. In: Proceedings of National Computer Conference, AFIPS, New York, pp. 313-317 (1979).

[2] Shamir, A.: How to share a secret. Commun. ACM 22, 612-613 (1979).

[3] Ingemarsson, I., Simmons, G. J.: A protocol to set up shared secret schemes without the assistance of a mutually trusted party. In: Advances in Cryptology - Proceedings of Eurocrypt'90, Springer, Berlin, pp. 266-282 (1991).

[4] Pedersen, T. P.: A threshold cryptosystem without a trusted party. In: Advances in Cryptology Proceedings of Eurocrypt'91, Springer, Berlin, pp. 522-526 (1991).

[5] Jackson, W.-A., Martin, K. M., O'Keefe, C. M.: Efficient secret sharing without a mutually trusted authority. In: Advances in Cryptology - Proceedings of Eurocrypt'95, Springer, Berlin, pp. 183-193 (1995).

[6] Hillery, M., Bǔzek, V., Berthiaume, A.: Quantum secret sharing. Phys. Rev. A 59, 1829-1834 (1999).

[7] Karlsson, A., Koashi, M., Imoto, N.: Quantum entanglement for secret sharing and secret splitting, Phys. Rev. A 59, 162-168 (1999).

[8] Cleve, R., Gottesman, D., Lo, H.-K.: How to share a quantum secret. Phys. Rev. Lett. 83, 648-651 (1999).

[9] Gottesman, D.: Theory of quantum secret sharing. Phys. Rev. A 61, 042311 (2000).

[10] Bandyopadhyay, S.: Teleportation and secret sharing with pure entangled states. Phys. Rev. A 62, $012308(2000)$.

[11] Nascimento, A. C. A., Mueller-Quade, J., Imai, H.: Improving quantum secret-sharing schemes. Phys. Rev. A 64, 042311 (2001). 
[12] Guo, G. P., Guo, G. C.: Quantum secret sharing without entanglement. Phys. Lett. A 310, 247-251 (2003).

[13] Lance, A. M., Symul, T., Bowen, W. P., Sanders, B. C., Lam, P. K.: Tripartite quantum state sharing. Phys. Rev. Lett. 92, 177903 (2004).

[14] Xiao, L., Long, G. L., Deng, F. G., Pan, J. W.: Efficient multiparty quantum-secret-sharing schemes, Phys. Rev. A 69, 052307 (2004).

[15] Tokunaga, Y., Okamoto, T., Imoto, N.: Threshold quantum cryptography. Phys. Rev. A 71, 012314 (2005).

[16] Zhang, Z. J., Li, Y., Man, Z. X.: Multiparty quantum secret sharing. Phys. Rev. A 71, 044301 (2005).

[17] Deng, F. G., Zhou, H. Y., Long, G. L.: Bidirectional quantum secret sharing and secret splitting with polarized single photons. Phys. Lett. A 337, 329-334 (2005).

[18] Takesue, H., Inoue, K.: Quantum secret sharing based on modulated high-dimensional time-bin entanglement. Phy. Rev. A 74, 012315 (2006).

[19] Zhang, Z. J.: Multiparty secret sharing of quantum information via cavity QED. Opt. Comm. 261, 199-202 (2006).

[20] Yu, I. C., Lin, F. L., Huang, C.Y.: Quantum secret sharing with multilevel mutually (un)biased bases. Phys. Rev. A 78, 012344 (2008).

[21] Wootters, W. K., Zurek, W. H.: A single quantum cannot be cloned. Nature 299, 802-803 (1982).

[22] Aigner, M., Ziegler, G. M.: Proofs from the book. Springer, Berlin, pp. 7-10 (2006).

[23] Crépeau, C., Gottesman, D., Smith, A.: Secure multi-party quantum computation. In: Proceedings of the 34th annual ACM symposium on Theory of computing, ACM, New York, pp. 643-652 (2002).

[24] Ben-Or, M., Crépeau, C., Gottesman, D., Hassidim, A., Smith, A.: Secure multiparty quantum computation with (only) a strict honest majority. In: Proceedings of 47th Annual IEEE Symposium on the Foundations of Computer Science, IEEE, Los Alamitos, pp. 249-260 (2006). 